\title{
Factores pronósticos y sobrevida a mediano plazo de una cohorte de pacientes con cáncer pulmonar atendidos en la red de salud de la Universidad Católica. Período 2007-2011
}

\author{
ARTURO MORALES S.*, CARLOS CALVO D.**, SERGIO GONZÁLEZ B.***, \\ ORLANDO DÍAZ P.* y FERNANDO SALDÍAS P.*
}

\section{Prognostic factors and short-term survival of a cohort of adult patients with lung cancer treated in a network of health, 2007-2011}

Lung cancer is the leading cause of death from malignancy worldwide. In Chile the magnitude of the problem and the diagnosis-associated survival are unknown. Methods: We examined a cohort of 202 adult patients with lung cancer histologically confirmed in a single health network between January 2007 and December 2011. We accessed to medical records and images files of patients, recording the clinical, histological, imaging and staging data. Patients were followed until December 2013 to assess survival. Results: The mean age of the cohort was $68.1 \pm 11.5$ years, $53 \%$ were male and $86 \%$ had a smoking history. $82.2 \%$ of the cases were symptomatic at diagnosis, been cough the symptom most frequently reported. The predominant histological subtype was adenocarcinoma (42\%), followed by squamous cell carcinoma (26.2\%). In women, adenocarcinoma was the leading histology variety $(56.4 \%)$, and in males it was adenocarcinoma (37\%) and squamous cell carcinoma (33.3\%). The majority of the patients were diagnosed at advanced stages of the disease. The 36-month survival rate was 46.1\%. The mean survival according to clinical stage was 70.7 month in stage I, 60.3 in stage II, 47.1 in IIIA, 12.3 in IIIB and 11.7 month in stage IV. According to histological variety, the mean survival was 36.6 month in adenocarcinoma, 33.8 in squamous cell carcinoma, 20.9 in large-cell carcinoma, 11.9 in small-cell carcinoma and 19.6 month in undifferentiated non small-cell carcinoma. There were no significant differences in survival by age or gender. Conclusion: The most common histological type was adenocarcinoma and short-term survival was related to the clinical staging and histological variants.

Key words: Lung neoplasm, histology, neoplasm staging, prognosis, survival, outcome.

\section{Resumen}

El cáncer pulmonar es la principal causa de muerte por neoplasia a nivel mundial. En Chile se desconoce la magnitud del problema y la sobrevida asociada al diagnóstico. Material y Métodos: Se examinó una cohorte de 202 pacientes adultos con cáncer pulmonar confirmados histopatológicamente en una red de salud entre Enero de 2007 y Diciembre de 2011. Se accedió a las fichas clínicas y archivos de imágenes de los pacientes, registrando las variables clínicas, histológicas, imagenológicas y la etapificación clínica. Se siguió prospectivamente a los pacientes hasta Diciembre de 2013 para determinar sobrevida. Resultados: La edad promedio de la cohorte fue de 68,1 $\pm 11,5$ años, 53\% eran varones y $86 \%$ tenía historia de tabaquismo. El 82,2\% de los casos presentaron síntomas al momento del diagnóstico, siendo la tos el más frecuente. La variedad histológica preponderante fue el adenocarcinoma (42\%), seguido del carcinoma escamoso (26,2\%). En las mujeres la mayoría de los tumores correspondieron a adenocarcinomas $(56,4 \%$ del total) y en varones predominaron el adenocarcinoma (37\%) y el carcinoma escamoso (33,3\%). La mayoría de los pacientes se diagnosticaron en estadios

* Departamento de Enfermedades Respiratorias, Facultad de Medicina, Pontificia Universidad Católica de Chile.

** Alumno de Medicina, Escuela de Medicina, Pontificia Universidad Católica de Chile.

***Departamento de Anatomía Patológica, Facultad de Medicina, Pontificia Universidad Católica de Chile. 
avanzados de la enfermedad. La sobrevida global a los 36 meses fue 46,1\%. La sobrevida media por estadio clínico fue de 70,7 meses en el estadio I, 60,3 meses en estadio II, 47,1 meses en IIIA, 12,3 meses en IIIB y 11,7 meses en IV. Según histología, la sobrevida media en meses fue de 36, 6 en adenocarcinoma, 33,8 en carcinoma escamoso, 20,9 en células grandes, 11,9 en células pequeñas y 19,6 en tumor no células pequeñas indiferenciado. No hubo diferencias significativas en la sobrevida por edad y género. Conclusión: La variedad histológica más frecuente es el adenocarcinoma y la sobrevida está relacionada a la etapificación clínica y variedad histológica.

Palabras clave: Neoplasia pulmonar, histología, etapificación, pronóstico, sobrevida.

\section{Introducción}

El cáncer de pulmón es la principal causa de muerte por neoplasia a nivel mundial ${ }^{1,2}$ y el tabaquismo constituye el principal factor asociado a su patogenia. Durante las últimas décadas en los países desarrollados se han registrado importantes cambios epidemiológicos en esta patología, siendo los más relevantes el aumento de la incidencia en el sexo femenino ${ }^{1}$, el aumento del subtipo histológico adenocarcinoma y la disminución del subtipo escamoso ${ }^{3}$; sin cambios significativos en el pronóstico a largo plazo $(17,5 \%$ de sobrevida a 5 años $)^{4}$.

En los países en vías de desarrollo la incidencia de neoplasias pulmonares continúa en aumento. En nuestro país, la tasa de mortalidad se elevó de $12,3 \times 10^{6}$ habitantes en el año 2000 a $15,6 \times$ $10^{6}$ en el 2010, representando la segunda causa de muerte por neoplasia en este periodo ${ }^{5}$. Para el año 2030 se proyecta un total de 12.000 muertes anuales por esta causa ${ }^{6}$.

La incidencia de cáncer de pulmón en nuestro país es desconocida. Se presume que irá en aumento, en forma paralela con el incremento continuo de la tasa de consumo de tabaco de la población, la que alcanza el $40,6 \%$ en la muestra poblacional de la Encuesta Nacional de Salud 2009-20107. Las incidencias de los diversos tipos histológicos de esta neoplasia son también desconocidas, sin poder por ende establecer tendencias en el tiempo.

Nos parece de suma importancia establecer sistemas de registros nacionales que permitan conocer la incidencia y prevalencia del cáncer de pulmón en la población, sus variedades histológicas, estadios de presentación, esquemas terapéuticos, factores de riesgo, uso de recursos sanitarios y sobrevida asociada. En consecuencia, el propósito de este estudio fue determinar el cuadro clínico, las variedades histológicas, los estadios clínicos y la sobrevida a mediano plazo de una cohorte de pacientes adultos con cáncer de pulmón atendidos en la red de salud de un hospital universitario docente entre los años 2007 y 2011.

\section{Material y Métodos}

Se examinó una cohorte de 202 pacientes adultos con cáncer pulmonar con confirmación histológica diagnosticados en la Red de Salud de la Pontificia Universidad Católica de Chile entre Enero de 2007 y Diciembre de 2011. Se accedió retrospectivamente a las fichas clínicas y archivos de imágenes de estos pacientes para registrar de forma protocolizada las variables clínicas y sociodemográficas consignadas en el momento del diagnóstico de la enfermedad, tales como: edad, sexo, lugar de procedencia, comorbilidades, consumo de tabaco y carga tabáquica, cuadro clínico (baja de peso, tos, expectoración, hemoptisis, dolor torácico, disnea), entre otros. Se revisaron las imágenes e informes radiográficos disponibles (radiografía de tórax, tomografía axial computarizada (TAC) de tórax, cintigrama óseo, tomografía de emisión de positrones (PETCT) de cuerpo entero, y resonancia magnética y/o TAC de cerebro), registrando las siguientes variables para la etapificación clínica: tamaño y localización del tumor $(\mathrm{T})$, compromiso ganglionar hiliar y mediastínico $(\mathrm{N})$, metástasis a distancia (M), presencia de derrame pleural, linfangitis carcinomatosa pulmonar, y nódulos pulmonares secundarios (registrando la ubicación de éstos últimos), junto a los valores estandarizados de captación máxima (Standardized Uptake Value, SUVmax.) del tumor en la PET-CT. El protocolo fue revisado y aprobado por el Comité de Ética de la Institución con exención de consentimiento informado.

El estudio histopatológico fue realizado en el Departamento de Anatomía Patológica de la P. Universidad Católica de Chile, consignando el subtipo histológico tumoral, y en las muestras quirúrgicas el tamaño tumoral patológico, el compromiso de los bordes quirúrgicos y estructuras adyacentes, el número de linfonodos analizados y la presencia de compromiso tumoral en ellos. El diagnóstico histológico fue realizado según la clasificación de la Organización Mundial de la Salud de $2004^{8}$. La información clínica e histopatológica nos permitió estadificar a los pacientes 
con tumores pulmonares de acuerdo a la séptima edición del sistema $\mathrm{TNM}^{9}$. Se siguió prospectivamente a los pacientes hasta Diciembre de 2013 para determinar su sobrevida, esta información fue obtenida directamente del registro civil del gobierno de Chile.

\section{Análisis estadístico}

Los resultados son expresados como valores promedio \pm desviación estándar para las variables medidas en escala numérica y en porcentaje para las medidas en escala nominal. Las variables cualitativas fueron comparadas mediante la prueba de $\chi^{2}$ y las variables continuas con la prueba $t$ de Student. Para examinar la sobrevida a mediano plazo se construyeron curvas de supervivencia de Kaplan-Meier, y para comparar la sobrevida de diferentes categorías de riesgo (edad, sexo, variedad histológica y etapificación clínica) se empleó la prueba log-rank. Se determinó la sobrevida a 36 meses ya que por metodología de inclusión progresiva entre 2007 y 2011 sólo un 46\% de los pacientes tenían 5 ó más años de seguimiento al finalizar el estudio. Para análisis de sobrevida por edad se dividió a la cohorte en cuartiles según rango etario. Para el análisis de sobrevida por histología sólo se incluyeron las variedades histológicas más frecuentes y por ende con mayor cantidad de casos. Las diferencias entre las variables fueron consideradas significativas con un valor de $\mathrm{p}<0,05$.

\section{Resultados}

Las características clínicas de la cohorte de 202 pacientes con cáncer pulmonar con confirmación histológica atendidos en la Red de Salud UC se muestran en la Tabla 1. La edad promedio fue 68,1 $\pm 11,5$ años (rango: 29 a 93 años), e incluyeron a 108 varones $(53,5 \%)$ y 94 mujeres $(46,5 \%)$. El $86 \%$ de la cohorte tenía antecedentes de tabaquismo, predominando entre los varones $(95,5 \%$ en hombres y $74 \%$ en mujeres), el consumo promedio de tabaco fue de 29,6 $\pm 24,2$ paquetes/año (rango: 0-140). El 82,2\% de los casos tenían síntomas respiratorios o sistémicos en el momento del diagnóstico, siendo los más frecuentes tos, dolor torácico, baja de peso y hemoptisis (Tabla 1). El 53\% de los pacientes con

Tabla 1. Características clínicas de la cohorte de pacientes adultos con diagnóstico de cáncer pulmonar atendidos en la Red de Salud de la Universidad Católica

\begin{tabular}{|lcc|}
\hline Características & $\mathbf{n}$ & \% \\
\hline $\mathrm{n}$ & 202 & Rango: $29-93$ \\
Edad & $68,1 \pm 11,5$ & $(53,5 \% / 46,5 \%)$ \\
Sexo (masculino/femenino) & $108 / 94$ & $(95,5 \% / 74 \%)$ \\
\% Fumadores (masculino/femenino) & & $51,7 \%$ \\
Manifestaciones clínicas* & & $29,7 \%$ \\
Tos & $61 / 118$ & $23,7 \%$ \\
Dolor torácico & $35 / 118$ & $23,7 \%$ \\
Baja de peso & $28 / 118$ & $20,3 \%$ \\
Hemoptisis & $28 / 118$ & $2,5 \%$ \\
Disnea & $24 / 118$ & $17,8 \%$ \\
Síntomas neurológicos & $3 / 118$ & Rango: $0,7-16$ \\
Ausencia de síntomas & $21 / 118$ & $56,1 \% / 43,9 \%$ \\
Manifestaciones radiográficas* & & Rango: $1-28,4$ \\
Tamaño tumoral (cm) & $4,71 \pm 3,0$ & $62,4 \%$ \\
Localización topográfica (D-I) & $92 / 72$ & $22,6 \%$ \\
Tumor (SUV máximo) & $10,0 \pm 6,9$ & $14,3 \%$ \\
Compromiso ganglionar & $88 / 141$ & $9,6 \%$ \\
Nódulos pulmonares secundarios & $26 / 115$ & $35,9 \%$ \\
Derrame pleural & $16 / 112$ & $53,9 \%$ \\
Linfangitis carcinomatosa & $11 / 114$ & $55 / 153$ \\
Metástasis & $103 / 191$ & \\
Letalidad a 36 meses & & \\
\hline Los datos se presentan con & & \\
\hline
\end{tabular}

Los datos se presentan como promedios \pm DE, número o porcentajes según corresponda. Abreviaturas: D: Pulmón derecho, I: Pulmón izquierdo, SUV: Standardized Uptake Value. *El denominador indica el número de pacientes cuya información estaba disponible para cada variable. Un paciente podría presentar más de un síntoma o signo radiográfico. 
tumores pulmonares diagnosticados en el período 2010-11 fueron etapificados mediante PET-CT de cuerpo entero. Un $22,6 \%$ de los pacientes presentaron nódulos pulmonares secundarios, de ellos 42,3\% estaban localizados en la región contralateral a la lesión primaria, $26,9 \%$ eran ipsilaterales pero ubicados en distinto lóbulo y $30,8 \%$ en el mismo lóbulo.

$\mathrm{Al}$ analizar los tipos histológicos del cáncer pulmonar en esta cohorte de 202 pacientes, observamos una marcada preponderancia de la variedad de adenocarcinoma (Tabla 2). Al revisar la tendencia en el tiempo observamos un incremento en la frecuencia de adenocarcinoma y carcinoma escamoso a lo largo del periodo analizado, manteniendo el primero siempre un predominio. El porcentaje de pacientes fumadores en las variedades adenocarcinoma y carcinoma escamoso fue de $84,6 \%$ y $87,2 \%$, respectivamente. En las mujeres la mayoría de los tumores correspondieron a adenocarcinomas $(56,4 \%$ del total para el género) y en varones predominaron las variedades histológicas de adenocarcinoma (37\%) y carcinoma escamoso $(33,3 \%)$.

Para la etapificación TNM clínica se revisaron los antecedentes clínicos y las imágenes radiográficas de los pacientes, clasificando su estadio clínico de acuerdo a TNM séptima edición?. En 171 de los 202 pacientes se pudo acceder a las imágenes radiográficas y al registro clínico completo que permitió una adecuada etapificación de los pacientes. De ellos, se excluyó a 16 pacientes con histología de células pequeñas, 3 con carcinoides típicos y 1 con la variedad mucoepidermoide. Por ende, en el análisis de estadio

Tabla 2. Variedades histológicas en 202 pacientes adultos con cáncer pulmonar atendidos en la Red de Salud de la Universidad Católica

\begin{tabular}{|lcc|}
\hline Variedades histológicas & n & \% \\
Adenocarcinoma & 85 & 42,0 \\
Carcinoma escamoso & 53 & 26,2 \\
Cáncer células pequeñas & 21 & 10,4 \\
No células pequeñas indiferenciado & 12 & 5,9 \\
Carcinoma bronquioloalveolar & 9 & 4,4 \\
Cáncer células grandes & 9 & 4,4 \\
Tumor carcinoide & 5 & 2,5 \\
Cáncer adenoescamoso & 3 & 1,5 \\
Carcinoma sarcomatoide & 2 & 1,0 \\
Cáncer neuroendocrino indiferenciado & 2 & 1,0 \\
Cáncer mucoepidermoide & 1 & 0,5 \\
\hline
\end{tabular}

clínico se incluyó a 151 pacientes. La mayoría de los pacientes debutaron en estadios avanzados de la enfermedad, siendo el porcentaje por estadio el siguiente: Etapa IA (15,9\%), IB (4,0\%), IIA $(10,6 \%)$, IIB $(3,3 \%)$, IIIA $(17,2 \%)$, IIIB $(13,9 \%)$ y IV $(35,1 \%)$. La presencia de síntomas respiratorios y sistémicos en el momento de la consulta fue más frecuente en los pacientes con estadios avanzados de la enfermedad $(92,7 \%$ de los pacientes en estadios III y IV versus $51,7 \%$ en los estadios I y II).

Del total de los pacientes incluidos en el estudio tuvimos seguimiento clínico en 191 pacientes, permitiéndonos analizar la sobrevida en el seguimiento a 36 meses, registrándose 103 fallecimientos, con una supervivencia actuarial de $46,1 \%$. Para el análisis de sobrevida por estadio TNM se incluyó sólo a los 151 pacientes en los que fue posible clasificar correctamente el estadio clínico con la información disponible. Dado el bajo número de casos en estadios IB y IIB, para el análisis de sobrevida según estadio TNM se consideró a estadios I como suma de IA+IB, y estadio II como suma de estadio IIA+IIB. La sobrevida media fue significativamente distinta según el estadio clínico de presentación $(70,7$ meses en estadio I; 60,3 meses en estadio II; 47,1 meses en IIIA; 12,3 meses en IIIB y 11,7 meses en IV; log-rank p <0,0001). Estas diferencias se explican principalmente por la mayor mortalidad observada en los estadios IIIB y IV en comparación a los otros grupos (Figura 1).

En el análisis de sobrevida según subtipo histológico, se descartaron las variedades histológicas de baja prevalencia, incluyendo en el análisis sólo a las más prevalentes. Por dicha razón el número de casos analizados en la sobrevida según subtipo histológico fue de 170 pacientes (Figura 2). La sobrevida media fue significativamente diferente para las diversos tipos histológicos (61,2 meses para bronquioloalveolar, 36,6 meses para adenocarcinoma, 33,8 meses para carcinoma escamoso, 20,9 meses en células grandes, 19,6 para el carcinoma no células pequeñas (NS-CLC) indiferenciado, y 11,9 meses para células pequeñas; $\log$-rank $\mathrm{p}<0,001)$. Las diferencias se deben principalmente a la peor sobrevida observada en pacientes con cáncer células pequeñas y NSCLC indiferenciado, en comparación a las otras variedades histológicas. Los pacientes en cuartil superior de edad (sobre 77 años) presentaron peor sobrevida que pacientes en cuartiles menores ( $\log$ rank $\mathrm{p}=0,008)$. Las mujeres tuvieron una mayor sobrevida media que los hombres (40,95 meses versus 28,15 meses, respectivamente; log rank $\mathrm{p}=0,011)$. 


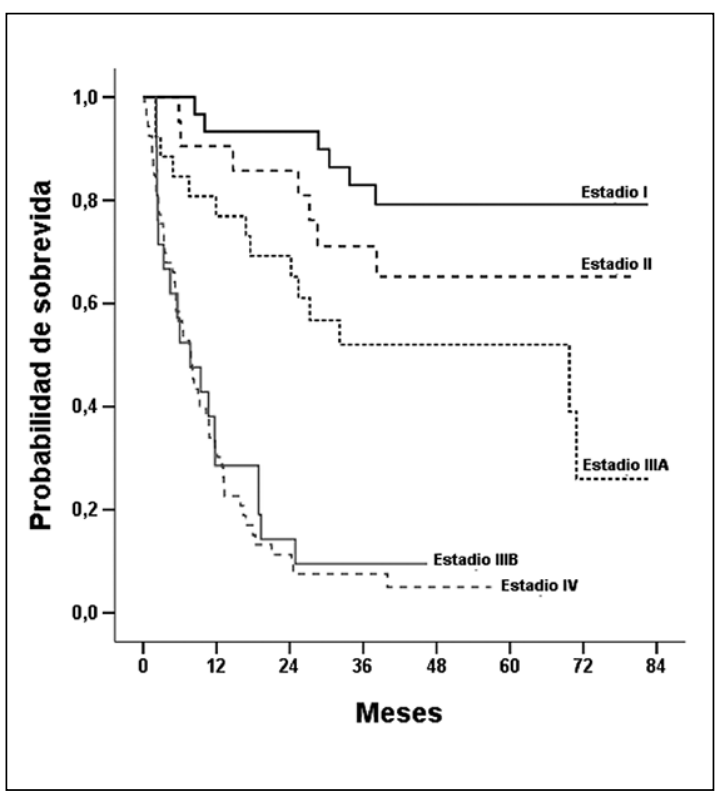

Figura 1. Sobrevida a mediano plazo de los pacientes con tumor pulmonar según estadio clínico (clasificación $\mathrm{TNM}^{9}$ ).

\section{Discusión}

En el estudio se describen las características clínico-radiológicas y patológicas de una cohorte de pacientes adultos con neoplasia pulmonar atendidos en una red de salud privada. La edad promedio de los pacientes incluidos en nuestra cohorte es similar a las edades de presentación de la neoplasia pulmonar en series extranjeras ${ }^{10,11}$, la mayoría son adultos mayores de 50 años fumadores. En nuestra serie la distribución de casos fue homogénea entre ambos sexos, lo que difiere de reportes internacionales donde, si bien la incidencia de cáncer de pulmón ha aumentado en los últimos años en mujeres, sigue siendo inferior a las tasas reportadas en hombres ${ }^{10,16}$. Esta diferencia pudiese ser atribuida a sesgos de medición y selección en el modelo del estudio; sin embargo, si consideramos las altas tasas de tabaquismo tanto en hombres como en mujeres en nuestro país $(37,1 \% \text { y } 44,2 \% \text {, respectivamente })^{7}$ podríamos plantear como hipótesis que la distribución por género de esta neoplasia a nivel nacional podría diferir de la reportada en series extranjeras. La elevada incidencia de tabaquismo en la cohorte, es similar a la reportada por autores extranjeros $(90 \%)^{12,14}$, lo que confirma la fuerte relación entre el consumo de tabaco y el cáncer de pulmón.

La presencia de síntomas respiratorios y sistémicos atribuibles a la enfermedad es más común en los estadios avanzadas de la enfermedad, lo

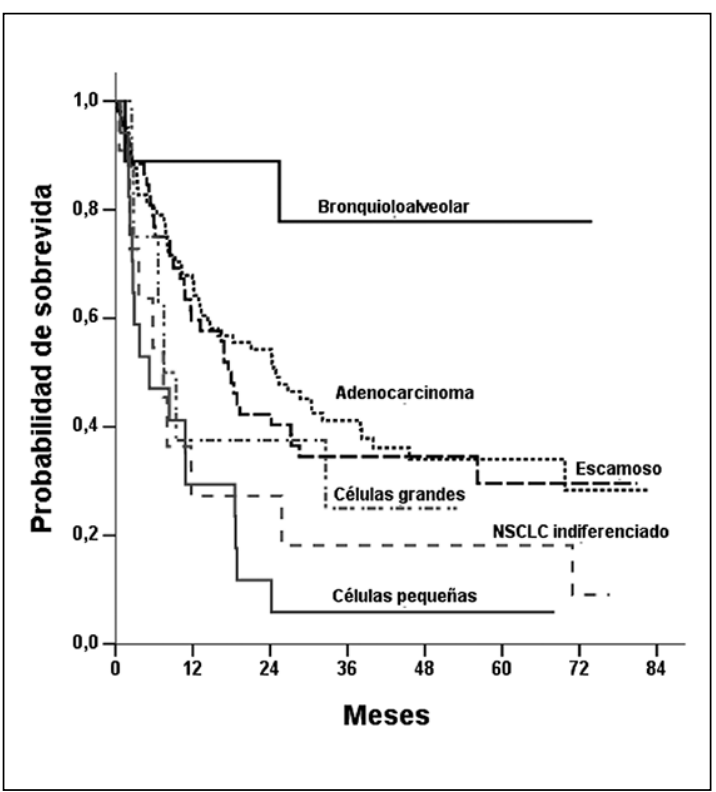

Figura 2. Sobrevida a mediano plazo de los pacientes con tumor pulmonar según variedad histológica. NSCLC: carcinoma no células pequeñas.

que dificulta el diagnóstico precoz. En nuestra serie el $92,7 \%$ de los pacientes diagnosticados en estadios III y IV presentaban algún síntoma, mientras que en estadios I y II sólo un $51,7 \%$. El diagnóstico en etapas avanzadas es uno de los grandes responsables de la baja sobrevida global atribuible a esta neoplasia ${ }^{15}$. Por este motivo, es necesario recurrir a técnicas diagnósticas de tamizaje más sensibles para la pesquisa precoz de esta condición, junto a estrategias de prevención y promoción de la cesación del hábito tabáquico en la población, para lograr reducir el riesgo y prolongar la sobrevida global de los pacientes con cáncer pulmonar.

El tipo histológico de adenocarcinoma fue el más frecuente en nuestra cohorte. Este predominio por sobre el carcinoma escamoso debe ser confirmado en estudios prospectivos. En los países desarrollados, se han reportado cambios históricos en la incidencia de los distintos tipos histológicos, disminuyendo las variedades escamosas y aumentando los adenocarcinomas; cambios que recién comienzan a apreciarse en países en vías de desarrollo ${ }^{3,10-13}$. Estas variaciones son atribuidas a cambios en el hábito tabáquico de la población, en el diseño de los cigarrillos, y en menor medida a mejores técnicas diagnósticas que facilitan la pesquisa precoz de nódulos periféricos ${ }^{3}$.

Un $66 \%$ de los casos de cáncer pulmonar fueron diagnosticados en estadios avanzados de la 
enfermedad (estadio III y IV), los que tuvieron peor sobrevida en el seguimiento, lo que concuerda con lo reportado previamente por otros autores internacionales ${ }^{10,15}$. Nos resulta sorprendente la alta sobrevida del estadio IIIA ( $52 \%$ a los 36 meses), a diferencia de las grandes series extranjeras que bordean el $28 \%{ }^{15}$. Esta diferencia puede estar relacionada a las características de la población, sesgos de selección y al pequeño tamaño muestral del presente estudio; sin embargo, diez de los 26 pacientes en estadio IIIA cumplían criterios de estadio IIIA en que se han asociado a mejor pronóstico (cuatro en T4N0, cuatro en $\mathrm{T} 3 \mathrm{~N} 1$ y dos en $\mathrm{T} 4 \mathrm{~N} 1$, en comparación a estadios que involucren N2) ${ }^{9}$. Las sobrevidas de los pacientes con variedades histológicas escamosas y adenocarcinoma fueron superiores a la observada en otras variedades histológicas, como el carcinoma de células pequeñas y el carcinoma de células grandes. En los tumores no células pequeñas indiferenciados, en los que no se pudo definir la clasificación definitiva por técnicas de inmunohistoquímica, la mortalidad fue similar a los tumores de células pequeñas.

La principal limitación del estudio fue el diseño descriptivo retrospectivo, lo que dificultó la obtención de la información clínico-radiológica y seguimiento clínico detallado, limitando la descripción de las características sociodemográficas, clínicas, imagenológicas, etapificación clínica (TNM), tratamiento implementado y evolución de los pacientes enrolados. Por otro lado, la inclusión de pacientes con cáncer pulmonar atendidos sólo en una red asistencial de salud de la Región Metropolitana limita la generalización de los resultados. La recolección de la información clínica de forma protocolizada por el equipo de investigadores junto a la revisión sistemática de las imágenes radiográficas e informes, permitió mejorar la calidad de la información obtenida al no recoger sólo los datos registrados en la ficha clínica. Por tratarse de un estudio clínico realizado en una red de salud privada, podría no reflejar la realidad de otros servicios de salud u otras áreas geográficas, por lo que se requieren de estudios de cohortes multicéntricos de mayor tamaño y que abarquen diferentes áreas geográficas para conocer en mayor profundidad la situación de este flagelo nacional.

\section{Bibliografía}

1.- SIEGEL R, NAISHADHAM D, JEMAL A. Cancer Statistics, 2013. CA Cancer J Clin 2013; 63: 11-30.

2.- ARFÈ A, MALVEZZI M, BERTUCCIO P, DECARLI
A, LA VECCHIA C, NEGRI E. Cancer mortality trend analysis in Italy, 1970-2007. Eur J Cancer Prev 2011; 20: 364-74.

3.- $\quad$ THUN M J, LALLY C A, FLANNERY J T, CALLE E E, FLANDERS W D, HEATH C W Jr. Cigarette smoking and changes in the histopathology of lung cancer. J Natl Cancer Inst 1997; 89: 1580-6.

4.- SEER (Surveillance, Epidemiology, and End Result Program). Surveillance Research Program. National Cancer Institute. 5 year relative survival for period 2003-2009 of diagnosis. http://seer.cancer.gov/csr/1975_2010/ browse_csr.php?sectionSEL $=15 \&$ pageSEL $=$ sect_15_table.12.html\#f

5.- MINISTERIO DE SALUD. Gobierno de Chile. Departamento de Estadísticas e Información de Salud. Serie de mortalidad por causa 2000-2009. http://deis.minsal. cl/vitales/Mortalidad causa/Chile.htm

6.- VALDIVIA G, BASTÍAS G. Epidemiología del cáncer en Chile. Boletín Esc. Medicina, Pontificia Universidad Católica de Chile 1994; 23: 45-9.

7.- MINISTERIO DE SALUD. Gobierno de Chile. Departamento de Epidemiología. Encuesta Nacional de Salud. Chile 2009-2010. http://epi.minsal.cl/wp-content/ uploads/2012/07/ Informe-ENS-2009-2010.-CAP-5 FINALv1julioccepi.pdf

8.- TRAVIS W D, BRAMBILLA E, NOGUCHI M, NICHOLSON A G, GEISINGER KR, YATABE Y, et al. International Association for the Study of Lung Cancer/ American Thoracic Society/ European Respiratory Society: International multidisciplinary classification of lung adenocarcinoma. J Thorac Oncol 2011; 6: 244-85.

9.- DETTERBECK F C, BOFFA D J, TANOUE L T. The new lung cancer staging system. Chest 2009; 136: 26071.

10.- GULLÓN J A, SUÁREZ I, MEDINA A, MARTÍN A, CABRERA C, GONZÁLEZ I J. Lung cancer: changes in epidemiology and survival. Rev Clin Esp 2012; 212: 18-23.

11.- MOUSAVI S M, SUNDQUIST K, HEMMINKI K. Risk of lung cancer by histology among immigrants to Sweden. Lung Cancer 2012; 76: 159-64.

12.- ALBERG A J, FORD J G, SAMET J M. American College of Chest Physicians. Epidemiology of lung cancer: ACCP evidence-based clinical practice guidelines $\left(2^{\text {nd }}\right.$ Edition). Chest 2007; 132 (3 Suppl): 29S-55S.

13.- SEER (Surveillance, Epidemiology and End Results Program), U.S. National Insitutes of Health, Cancer Statistics Review, Percent Distribution and Counts by Histology among Histologically Confirmed Cases, 2005-2009. Disponible en: http://seer. cancer.gov/csr/1975_2009_pops09/browse_csr.php? section $=15 \&$ page $=$ sect 15 table $.28 . \mathrm{html}$

14.- JIMÉNEZ MASSA A E, ALONSO SARDÓN M, GÓMEZ GÓMEZ F P. Lung cancer: how does it appear in our hospital? Rev Clin Esp 2009; 209: 110-7.

15.- GOLDSTRAW P, CROWLEY J, CHANSKY K, GI- 
ROUX D J, GROOME P A, RAMI-PORTA R, et al. The IASLC Lung Cancer Staging Project: proposals for the revision of the TNM stage groupings in the forthcoming (seventh) edition of the TNM Classification of malignant tumours. J Thorac Oncol 2007;2:706-14.
16.- JEMAL A, THUN M J, RIES L A, HOWE H L, WEIR $\mathrm{H}$ K, CENTER M M, et al. Annual report to the nation on the status of cancer, 1975-2005, featuring trends in lung cancer, tobacco use, and tobacco control. J Natl Cancer Inst 2008; 100: 1672-94.

Correspondencia a:

Dr. Arturo Morales Soto

Departamento de Enfermedades Respiratorias Facultad de Medicina - División de Medicina Pontificia Universidad Católica de Chile

Marcoleta 350 - Primer Piso

Santiago - Chile

Fono: 223543242 / Celular 9-5099041

Fax: 226335255

Email: ajmorals@gmail.com 